\title{
Spectral Properties of Schrödinger-type Operators and Large-time Behavior of the Solutions to the Corresponding Wave Equation
}

\author{
A.G. Ramm * \\ Department of Mathematics, Kansas State University, Manhattan, KS 66506-2602, USA
}

Abstract. Let $L$ be a linear, closed, densely defined in a Hilbert space operator, not necessarily selfadjoint. Consider the corresponding wave equations

$$
\begin{aligned}
& \text { (1) } \ddot{w}+L w=0, \quad w(0)=0, \quad \dot{w}(0)=f, \quad \dot{w}=\frac{d w}{d t}, \quad f \in H . \\
& \text { (2) } \ddot{u}+L u=f e^{-i k t}, \quad u(0)=0, \quad \dot{u}(0)=0,
\end{aligned}
$$

where $k>0$ is a constant. Necessary and sufficient conditions are given for the operator $L$ not to have eigenvalues in the half-plane $\operatorname{Re} z<0$ and not to have a positive eigenvalue at a given point $k_{d}^{2}>0$. These conditions are given in terms of the large-time behavior of the solutions to problem (1) for generic $f$.

Sufficient conditions are given for the validity of a version of the limiting amplitude principle for the operator $L$.

A relation between the limiting amplitude principle and the limiting absorption principle is established.

Keywords and phrases: elliptic operators, wave equation, limiting amplitude principle, limiting absorption principle

Mathematics Subject Classification: 35P25, 35L90, 43A32

\section{Introduction}

Let $L$ be a linear, densely defined, closed operator in a Hilbert space $H$. Our results and techniques are valid in a Banach space also, but we wish to think about $L$ as of a Schrödinger-type operator in a Hilbert space and, at times, think that $L$ is selfadjoint. For a Schrödinger operator $L=-\nabla^{2}+q(x)$ the resolvent $\left(L-k^{2}\right)^{-1}, I m k>0$, is an integral operator with a kernel $G(x, y, k)$, its resolvent kernel. If $q$ is a real-valued function, sufficiently rapidly decaying then $L$ is selfadjoint, $G(x, y, k)$ is analytic with respect to $k$ in the half-plane $\operatorname{Im} k>0$, except, possibly, for a finitely many simple poles $i k_{j}, k_{j}>0$, the

\footnotetext{
${ }^{*}$ Corresponding author. E-mail: ramm@math.ksu.edu
} 
semiaxis $k \geq 0$ is filled with the points of absolutely continuous spectrum of $L$, and there exists a limit

$$
\lim _{\epsilon \rightarrow 0} G(x, y, k+i \epsilon)=G(x, y, k)
$$

for all $k>0$.

Sufficient conditions for $k^{2}=0$ not to be an eigenvalue of $L$ are found in papers [5], [6]. Spectral analysis of the Schrödinger operators is presented in many books (see, for example, [2] and [11]). In papers [3], [4], such an analysis was given in a class of domains with infinite boundaries apparently for the first time, see also [8]. In [7] an eigenfunctions expansion theorem was proved for non-selfadjoint Schrödinger operators with exponentially decaying complex-valued potential $q$. The operator $L$ in this paper is not necessarily assumed to be selfadjoint.

In [1] the validity of the limiting amplitude principle for some class of selfadjoint operators $L$ has been established.

This principle says that, as $t \rightarrow \infty$, the solution to problem

$$
\ddot{u}+L u=f e^{-i k t}, \quad u(0)=0, \quad \dot{u}(0)=0, \quad \dot{u}=\frac{d u}{d t},
$$

has the following asymptotics

$$
u=e^{-i k t} v+o(1), \quad t \rightarrow \infty,
$$

where $k$ is a real number and $v \in H$ solves the equation

$$
L v-k^{2} v=f .
$$

The $v$ is called the limiting amplitude. It turns out that a more natural definition of the limiting amplitude is:

$$
v=\lim _{t \rightarrow \infty} \frac{1}{t} \int_{0}^{t} u(s) e^{i k s} d s,
$$

if this limit exists and solves equation (1.3).

Why is this definition more natural than (1.2)? There are good reasons for this. One of the reasons is: if (1.2) and (1.3) hold, then the limit (1.4) exists and solves equation (1.3). The other reason is: the limit (1.4) may exist and solve equation (1.3) although the limit (1.2) does not exist.

Example. If $u=e^{i k t} v+e^{i k_{1} t} v_{1}$, then the limit (1.2) does not exist, while the limit (1.4) does exist and is equal to $v$.

To describe our assumptions and results, some preparation is needed.

Consider the problem

$$
\ddot{w}+L w=0, \quad w(0)=0, \quad \dot{w}(0)=f .
$$

Assuming that $\|u(t)\| \leq c e^{a t}$, where $c>0$ stands throughout the paper for various generic constants, and $a \geq 0$ is a constant, one can define the Laplace transform of $u(t)$,

$$
\mathcal{U}:=\mathcal{U}(p):=\int_{0}^{\infty} e^{-p t} u(t) d t, \quad \sigma>a,
$$

where $p=\sigma+i \tau, \operatorname{Re} p=\sigma$.

Let us take the Laplace transform of (1.1) and of (1.5) to get

$$
L \mathcal{U}+p^{2} \mathcal{U}=\frac{f}{p+i k},
$$

and

$$
L \mathcal{W}+p^{2} \mathcal{W}=f
$$


where

$$
\mathcal{W}=\mathcal{W}(p)=\int_{0}^{\infty} w(t) e^{-p t} d t
$$

We also denote $\mathcal{W}(p):=\bar{w}(t)$.

The complex plane $p$ is related to the complex plane $k$ by the formula

$$
p=-i k, \quad k=k_{1}+i k_{2}, \quad k_{2} \geq 0, \quad \sigma=k_{2} \geq 0 .
$$

We assume throughout that $f$ is generic in the following sense:

If $I$ is the identity operator and a point $p$ is a pole of the kernel of the operator $\left(L+p^{2} I\right)^{-1}$, then it is a pole of the same order of the element $\left(L+p^{2} I\right)^{-1} f=\mathcal{W}$.

If $k^{2}$ is an eigenvalue of $L$ and $\operatorname{Re} k^{2}<0$, then $\operatorname{Im} k>0$, where $k=|k| e^{\frac{i \arg k^{2}}{2}}, p=-i k$, so $\sigma=\operatorname{Re} p>0$. Let $k>0$ and assume that $-k^{2}<0$ is an eigenvalue of $L$. Then $i k$ is a pole of the resolvent kernel $G(x, y, k)$, and $p=-i(i k)=k$ is a pole of the kernel of the operator $\left(L+p^{2} I\right)^{-1}$. If $k^{2}>0$ is an eigenvalue of $L$, then $p=-i k$ is a pole of the operator $\left(L+p^{2} I\right)^{-1}$.

The following known facts from the theory of Laplace transform will be used.

Proposition 1.1. An analytic in the half-plane $\sigma>\sigma_{0} \geq 0$ function $F(p)$ is the Laplace transform of a function $f(t)$, such that $f(t)=0$ for $t<0$ and

$$
\int_{0}^{\infty}|f(t)|^{2} e^{-2 \sigma_{0} t} d t<\infty
$$

if and only if

$$
\sup _{\sigma>\sigma_{0}} \int_{-\infty}^{\infty}|F(\sigma+i \tau)|^{2} d \tau<\infty
$$

Proposition 1.2. If $F(p)=\overline{f(t)}$, then

$$
\frac{F(p)}{p}=\overline{\int_{0}^{t} f(s) d s .}
$$

Let us now formulate the main Assumptions A and B standing throughout this paper.

Assumption A. For a generic $f$ the $\mathcal{W}(p)=\left(L+p^{2}\right)^{-1} f$ is analytic in the half-plane $\sigma>0$, except, possibly, at a finitely many simple poles at the points $-i k_{j}, 1 \leq j \leq J, k_{j}$ are real numbers, and at the points $\kappa_{m}$, Re $\kappa_{m}>0$,

$$
\mathcal{W}(p)=\sum_{j=1}^{J} \frac{v_{j}}{p+i k_{j}}+\mathcal{W}_{1}(p)+\sum_{m=1}^{M} \frac{b_{m}}{p-\kappa_{m}},
$$

where $v_{j}$ and $b_{m}$ are some elements of $H, \mathcal{W}_{1}(p)$ is an analytic function in the half-plane $\operatorname{Re} p=\sigma>0$, continuous up to the imaginary axis $\sigma=0$, and satisfying the following estimate

$$
\left\|\mathcal{W}_{1}(p)\right\| \leq \frac{c}{1+|p|^{\gamma}}, \quad \gamma>\frac{1}{2} .
$$

Assumption B. There exists the limit

$$
\lim _{\sigma \rightarrow 0}\left\|\mathcal{W}_{1}(\sigma-i k)-\mathcal{W}_{1}(-i k)\right\|=0
$$

for all real numbers $k$. 
Theorem 1.3. Let the Assumption A hold. Then a necessary and sufficient condition for the operator $L$ to have no eigenvalues in the half-plane $\operatorname{Re} k^{2}<0$ is the validity of the estimate

$$
\left\|\int_{0}^{t} w(s) d s\right\|=O\left(e^{\epsilon t}\right), \quad t \rightarrow \infty
$$

for an arbitrary small $\epsilon>0$.

A necessary and sufficient condition for the operator $L$ not to have any positive eigenvalues $k^{2}>0$ is the validity of the estimate

$$
\left\|\frac{1}{t} \int_{0}^{t} e^{i k s} w(s) d s\right\|=o(1), \quad t \rightarrow \infty, \quad \forall k \in \mathbb{R} .
$$

A point $i k_{0}>0, k_{0}>0$, is not a pole of the resolvent kernel of the operator $\left(L-k^{2}-i 0\right)^{-1}$ if and only if estimate (1.16) holds with $k=k_{0}>0$.

Remark. If condition (1.16) holds for $k=0$, then $\left\|\int_{0}^{t} w(s) d s\right\|=o(t)$, so condition (1.15) holds, and the operator $L$ has no eigenvalues in the half-plane $\operatorname{Re} k^{2}<0$.

Theorem 1.4. Let the Assumptions $A$ and B hold. Suppose that estimates (1.14) and (1.15) hold. Then the limiting amplitude principle (1.4) holds for every $k \in \mathbb{R}, k \neq k_{j}, 1 \leq j \leq J$.

In section 2, proofs are given.

\section{Proofs}

\subsection{Proof of Theorem 1.3}

From the Assumption A and Proposition 1.1, it follows that $\mathcal{W}(p)$ is a Laplace transform of a function $w(t)$ such that

$$
w(t)=\sum_{j=1}^{J} v_{j} e^{-i k_{j} t}+\sum_{m=1}^{M} b_{m} e^{\kappa_{m} t}+w_{1}(t),
$$

where

$$
w_{1}(t)=\frac{1}{2 \pi i} \int_{\sigma_{0}-i \infty}^{\sigma_{0}+i \infty} e^{p t} \mathcal{W}_{1}(p) d p,
$$

and the integral in (2.2) converges in $L^{2}$-sense due to the assumption (1.13). It is clear from formula (2.1) that all $b_{m}=0$ if and only if estimate (1.15) holds with $0<\epsilon<\min _{1 \leq m \leq M} \operatorname{Re} \kappa_{m}$. This proves the first conclusion of Theorem 1.3.

Let us calculate the expression on the left side of formula (1.16) and show that this expression is $o(1)$ unless $k=k_{j}$ for some $1 \leq j \leq J$. In this calculation it is assumed that $L$ does not have any eigenvalues in the half-plane Re $k^{2}<0$, in other words, that all $b_{m}=0$. Otherwise the expression on the left of formula (1.16) tends to infinity as $t \rightarrow \infty$ at an exponential rate.

If all $b_{m}=0$ in $(2.1)$, then

$$
\sum_{j=1}^{J} v_{j} \frac{1}{t} \int_{0}^{t} e^{i\left(k-k_{j}\right) t} d t+\frac{1}{t} \int_{0}^{t} w_{1}(t) e^{i k t} d t:=I_{1}+I_{2} .
$$

If $k$ and $k_{j}$ are real numbers, then

$$
\lim _{t \rightarrow \infty} \frac{1}{t} \int_{0}^{t} e^{i\left(k-k_{j}\right) t} d t= \begin{cases}1, & k=k_{j} \\ 0, & k \neq k_{j} .\end{cases}
$$


Thus, $I_{1}=0$ if and only if $k$ does not coincide with any of $k_{j}, 1 \leq j \leq J$.

Let us prove that

$$
\lim _{t \rightarrow \infty} \frac{1}{t} \int_{0}^{t} w_{1}(t) e^{i k t} d t=0 .
$$

By proposition (1.2) and the Mellin inversion formula, one has

$$
I:=\frac{1}{t} \int_{0}^{t} w_{1}(t) e^{i k t} d t=\frac{1}{2 \pi i} \int_{\sigma-i \infty}^{\sigma+i \infty} \mathcal{W}_{1}(p-i k) \frac{e^{p t}}{p t} d p
$$

where $\operatorname{Re} p=\sigma>0$ can be chosen arbitrarily small.

Let $p t=q$, take $\sigma=\frac{1}{t}$, write $q=1+i s$, and write the integral on the right side of (2.6) as:

$$
I=\frac{1}{2 \pi i} \int_{1-i \infty}^{1+i \infty} \mathcal{W}_{1}\left(\frac{q}{t}-i k\right) \frac{q}{t} \frac{e^{q}}{q^{2}} d q
$$

If one uses estimate (1.13) and formula $|q|=\left(1+s^{2}\right)^{1 / 2}$, then one obtains the following inequality

$$
\|I\| \leq \frac{1}{2 \pi t} \int_{-\infty}^{\infty} \frac{1}{\left(1+s^{2}\right)^{1 / 2}} \frac{c d s}{\left[1+\left|\frac{1+i s}{t}-i k\right|^{\gamma}\right]}=\frac{c}{2 \pi t^{1-\gamma}} \int_{-\infty}^{\infty} \frac{1}{\left(1+s^{2}\right)^{1 / 2}} \frac{d s}{\left(t^{\gamma}+\left[1+(s-k t)^{2}\right]^{\gamma / 2}\right)} .
$$

Let $s=t y$. Then the integral on the right side of (2.8) can be written as

$$
\begin{aligned}
& \frac{c t}{2 \pi t^{1-\gamma}} \int_{-\infty}^{\infty} \frac{d y}{\left(1+t^{2} y^{2}\right)^{1 / 2}} \frac{1}{\left(t^{\gamma}+\left[1+t^{2}(y-k)^{2}\right]^{\gamma / 2}\right)} \\
& =\frac{c}{2 \pi} \int_{-\infty}^{\infty} \frac{d y}{\left(1+t^{2} y^{2}\right)^{1 / 2}} \frac{1}{\left(1+\left[t^{-2}+(y-k)^{2}\right]^{\gamma / 2}\right)} \\
& \leq \frac{c}{2 \pi} \int_{-\infty}^{\infty} \frac{d y}{\left(1+t^{2} y^{2}\right)^{1 / 2}} \frac{1}{\left[1+(y-k)^{\gamma}\right]} \rightarrow 0, \text { as } t \rightarrow \infty,
\end{aligned}
$$

and the convergence of the last integral to zero is uniform with respect to $k \in \mathbb{R}$.

Thus

$$
\lim _{t \rightarrow \infty}\|I\|=0 .
$$

From (2.3)-(2.5) the last two conclusions of Theorem 1.3 follow. Theorem 1.3 is proved.

\subsection{Proof of Theorem 1.4}

Using Proposition 1.2, equation (1.6), and the Mellin formula, one gets

$$
\frac{1}{t} \int_{0}^{t} u(t) e^{i k t} d t=\frac{1}{t} \frac{1}{2 \pi i} \int_{\sigma-i \infty}^{\sigma+i \infty} \frac{\mathcal{U}(p-i k)}{p} e^{p t} d p
$$

where, according to (1.6),

$$
\mathcal{U}(p-i k)=\frac{\mathcal{W}(p-i k)}{p}
$$

Let $\sigma=\frac{1}{t}$ and $p t=q$. Then

$$
\frac{1}{t} \int_{0}^{t} u(t) e^{i k t} d t=\frac{1}{2 \pi i} \int_{1-i \infty}^{1+i \infty} \mathcal{W}\left(\frac{q}{t}-i k\right) \frac{e^{q}}{q^{2}} d q
$$

Estimate (1.15) and Theorem 1.3 imply that all $b_{m}=0$ in formula (2.1). Therefore, using formula (2.1) with $b_{m}=0$, one gets

$$
\mathcal{W}=\sum_{j=1}^{J} v_{j} \frac{1}{p+i k_{j}}+\mathcal{W}_{1}
$$


and

$$
\mathcal{W}\left(\frac{q}{t}-i k\right)=\mathcal{W}_{1}\left(\frac{q}{t}-i k\right)+\sum_{j=1}^{J} v_{j} \frac{1}{\frac{q}{t}-i\left(k-k_{j}\right)} .
$$

One has $\overline{t^{n}}=\frac{n !}{p^{n+1}}$. Therefore

$$
\frac{1}{2 \pi i} \int_{1-i \infty}^{1+i \infty} \frac{e^{q}}{q^{2}} d q=1
$$

and

$$
\lim _{t \rightarrow \infty} \frac{v_{j}}{2 \pi i} \int_{1-i \infty}^{1+i \infty} \frac{1}{\frac{q}{t}-i\left(k-k_{j}\right)} \frac{e^{q}}{q^{2}} d q= \begin{cases}\frac{i v_{j}}{k-k_{j}}, & k \neq k_{j} \\ \infty, & k=k_{j}\end{cases}
$$

Furthermore,

$$
\lim _{t \rightarrow \infty} \frac{1}{2 \pi i} \int_{1-i \infty}^{1+i \infty} \mathcal{W}_{1}\left(\frac{q}{t}-i k\right) \frac{e^{q}}{q^{2}} d q=\mathcal{W}_{1}(-i k)
$$

as follows from assumption (1.14) and the Lebesgue's dominated convergence theorem if one passes to the limit $t \rightarrow \infty$ under the sign of the integral (2.16). Let us check that this $v$ solves equation (1.3). This would conclude the proof of Theorem 1.4. We need a lemma.

Lemma 2.1. If $h \in L_{l o c}^{1}(0, \infty)$ and the limit $\lim _{t \rightarrow \infty} t^{-1} \int_{0}^{t} h(s) d s$ exists, then the limit $\lim _{p \rightarrow 0} p \int_{0}^{\infty} e^{-p t} h(t) d t$ exists, and

$$
\lim _{t \rightarrow \infty} t^{-1} \int_{0}^{t} h(s) d s=\lim _{p \rightarrow 0} p \int_{0}^{\infty} e^{-p t} h(t) d t .
$$

Proof of Lemma 1. One has

$$
p \int_{0}^{\infty} e^{-p t} h(t) d t=\left.p e^{-p t} \int_{0}^{t} h(s) d s\right|_{0} ^{\infty}+p^{2} \int_{0}^{\infty} t e^{-p t} t^{-1} \int_{0}^{t} h(s) d s d t .
$$

For any $p>0$ one has

$$
\left.p e^{-p t} \int_{0}^{t} h(s) d s\right|_{0} ^{\infty}=0 .
$$

Let $q=p t$ and denote $H(t):=t^{-1} \int_{0}^{t} h(s) d s, J:=\lim _{t \rightarrow \infty} H(t)$. Then

$$
\lim _{p \rightarrow 0} p^{2} \int_{0}^{\infty} t e^{-p t} t^{-1} \int_{0}^{t} h(s) d s d t=\lim _{p \rightarrow 0} \int_{0}^{\infty} q e^{-q} H\left(q p^{-1}\right) d q .
$$

Passing in the last integral to the limit $p \rightarrow 0$ one obtains (2.17). Lemma 1 is proved.

Using equation (2.17), one writes $v=\lim _{p \rightarrow 0} p \mathcal{U}(p-i k)$, where $\mathcal{U}$ solves equation (1.6). Thus,

$$
L \mathcal{U}(p-i k)+(p-i k)^{2} \mathcal{U}(p-i k)=p^{-1} f .
$$

Multiplying both sides of this equation by $p$ and passing to the limit $p \rightarrow 0$, one obtains equation (1.3). In the passage to the limit under the sign of the unbounded operator $L$ the assumption that $L$ is closed was used.

Thus, the conclusion of Theorem 1.4 follows.

If the limit (1.14) exists at a point $p=i \tau$ then one says that the limiting absorption principle holds for the operator $L$ at the point $k=i p=i(-i k)=k, k>0$.

Thus, Assumption B means that the limiting absorption principle holds for $L$ at the point $k>0$, that is, $\lim _{\epsilon \rightarrow 0}\left(L-k^{2}-i \epsilon\right)^{-1} f$ exists. 


\section{Applications}

Let $L=-\nabla^{2}+q(x)$, where $q(x)$ is a real-valued function, $|q(x)| \leq c(1+|x|)^{-2-\epsilon}, \epsilon>0, x \in \mathbb{R}^{3}$. Then $L$ is selfadjoint on the domain $H^{2}\left(\mathbb{R}^{3}\right)$. Its resolvent $\left(L-k^{2}-i 0\right)^{-1}$ satisfies Assumptions $\mathrm{A}$ and $\mathrm{B}$ if one keeps in mind the following.

Let $G(x, y, k)$ be the resolvent kernel of $L$, that is, the kernel of the operator $\left(L-k^{2}-i 0\right)^{-1}$,

$$
L G(x, y, k)=-\delta(x-y) \text { in } \mathbb{R}^{3},
$$

$G \in L^{2}\left(\mathbb{R}^{3}\right)$ for $\operatorname{Im} k>0$. If $f \in L^{2}\left(\mathbb{R}^{3}\right)$ is compactly supported, then for $k>0$ the function

$$
v(x):=\left(L-k^{2}-i 0\right)^{-1} f=\int_{\mathbb{R}^{3}} G(x, y, k) f(y) d y
$$

does not necessarily belong to $L^{2}\left(\mathbb{R}^{3}\right)$.

For example, if $q(x)=0$, then $G(x, y, k)=\frac{e^{i k|x-y|}}{4 \pi|x-y|}$, and the function

$$
v(x, k)=\int_{|y| \leq 1} g(x, y, k) d y=O\left(\frac{1}{|x|}\right)
$$

does not belong to $L^{2}\left(\mathbb{R}^{3}\right)$ (except for those $k>$ for which $x(x, k)=0$ in the region $|y| \geq 1$. These numbers $k>0$ are the zeros of the Fourier transform of the characteristic function of the ball $|y| \leq 1$, see [10], Chapter 11.

By this reason the abstract results of theorem (1.3) and (1.4) can be used in applications if one defines some subspace of $H$, for example, a subspace of functions with compact support, denote by $\mathcal{P}$, a projection operator on this subspace, and replaces $\mathcal{W}$ and $\mathcal{W}_{1}$ by $\mathcal{P W}$ and $\mathcal{P} \mathcal{W}_{1}$ in equations (1.12) and (1.14). For example, the function (3.1) one replaces by $\eta(x) v(x, k)$, where $\eta(x)$ is a characteristic function of a compact subset of $\mathbb{R}^{3}$.

The analytic properties of $\eta(x) v(x, k)$ and of $v(x, k)$ as functions of $k$ are the same. A similar suggestion is used in [1].

With the above in mind, one knows (for example, from [2] or [11]) that Assumptions A and B hold for $L=-\nabla^{2}+q(x)$.

Consequently, the conclusions of Theorems 1.3 and 1.4 hold.

In addition, the assumptions

$$
|q(x)| \leq c(1+|x|)^{-2-\epsilon}, \quad \epsilon>0, \quad \operatorname{Im} q=0,
$$

imply that $L$ does not have positive eigenvalues, so all $v_{j}=0$, and zero is not an eigenvalue of $L \geq 0$ if $\epsilon>0$ (see [5], [6]).

A new method for estimating of large time behavior of solutions to abstract evolution problems is developed in [9], where some applications of this method are given.

\section{References}

[1] D. Eidus. The limiting amplitude principle. Russ. Math. Surveys, 24, (1969), 51-94

[2] D. Pearson. Quantum scattering and spectral theory. Acad. Press, London, 1988.

[3] A.G. Ramm. Spectral properties of the Schrödinger operator in some domains with infinite boundaries. Doklady Acad of Sci. USSR, 152, (1963) 282-285.

[4] A.G. Ramm. Spectral properties of the Schrödinger operator in some infinite domains. Mat. Sborn., 66, (1965), 321-343.

[5] A.G.Ramm. Sufficient conditions for zero not to be an eigenvalue of the Schrödinger operator. J. Math. Phys., 28, (1987), 1341-1343.

[6] A.G.Ramm. Conditions for zero not to be an eigenvalue of the Schrödinger operator. J. Math. Phys. 29, (1988), 1431-1432.

[7] A.G. Ramm. Eigenfunction expansion for nonselfadjoint Schrödinger operator. Doklady Acad. Sci. USSR. 191, (1970), 50-53. 
[8] A.G. Ramm. Scattering by obstacles. D.Reidel, Dordrecht, 1986.

[9] A.G. Ramm.Stability of solutions to some evolution problems. Chaotic Modeling and Simulation (CMSIM), 1, (2011), 17-27.

[10] A.G. Ramm. Inverse problems. Springer, New York, 2005.

[11] M. Schechter. Operator methods in quantum mechanics. North Holland, New York, 1981. 